\title{
EDITORIAL
}

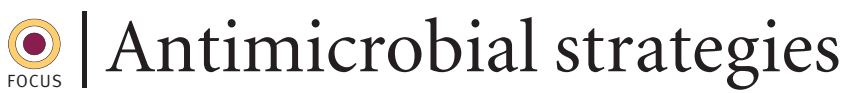

\section{STAYING AHEAD OF THE FOURTH HORSEMAN}

John Rex advocates strong public leadership in the drive to develop new anti-infectives.

Although the classical horsemen of the Apocalypse (Conquest, Strife, Famine and Pestilence) have historically proven remarkably persistent, society has made progress against them all. Pestilence in particular has yielded to our efforts - penicillin and other antibiotics have, when taken with other medical advances, radically altered our view on expected levels of health and longevity.

Unfortunately, some long-standing flaws in our defences against Pestilence have recently begun to enlarge. Multidrug-resistant microorganisms progressively reduce our options both in the hospital and at home. Bioterrorism threatens and genetic engineering could render pathogens even more resistant. And, of course, we still lack truly effective agents for some diseases. It is partly for these reasons that policy-level activity in support of the discovery of new anti-infectives has recently emerged.

Although we might yet squeeze a bit more activity from known drug classes, the discovery of therapies with new modes of action is the best way to bypass known resistance mechanisms. The echinocandin antifungals introduced during the 1990s are good examples of this. Amphotericin B and the azoles had long provided effective anti-Candida therapy, but were limited by toxicity and resistance, respectively. Although the echinocandins also have limitations, their different safety and efficacy profiles make them most welcome.

How should we continue to seek novel strategies? Articles in this issue illustrate the two possible general approaches. Narrow-spectrum tools such as antitoxins, antibodies and anti-pathogenesis tools are intriguing. But, these tools require firm diagnoses, thereby limiting them to diseases amenable to vaccination, the few diseases with quick and accurate diagnostics, rapidly progressing epidemic situations, and chronic infections such as HIV, hepatitis B and C, tuberculosis or cryptococcosis.

Broad-spectrum agents suitable for reliable empirical therapy are the goal of most projects, especially in the antibacterial arena. Unfortunately, novel classes have so far been rare. Genomic-based target selection seems a logical way to fill this gap but is only now beginning to bear fruit. The key to success seems to lie in the effective use of a range of lead-generation tools - excessive reliance on simple screening of molecules present in corporate compound libraries appears inadequate.

But, this excellent science comes at a cost. And, as failure is the most likely outcome for any particular discovery programme, it is well that our market-based economies can provide incentives that encourage variety and persistence. Recently estimated at US \$802 million per new molecule (fully capitalized cost in 2000 dollars), discovery and development costs have outpaced inflation ${ }^{1}$. Investment of such staggering sums in the hope of a return 5-10 years later requires nerves of steel!

This combination of cost and delayed return represents the biggest threat to discovery of new agents. This issue of Nature Reviews Microbiology shows we have the tools but will business invest? Current worldwide controversies surrounding drug pricing, variations in regulatory requirements and increased regulatory challenges for all drugs are driving companies to critically reassess their plans. Companies may view anti-infectives as producing a lower return on limited capital (in large part due to their usually short-term use) and choose to pull out from at least some parts of this area ${ }^{2}$. And, incentives to develop therapies for diseases with limited market pull are even more elusive.

As Pestilence advances relentlessly, the prospect of reduced discovery efforts is frightening. It takes years to make progress - teams must gain experience and momentum before success becomes likely. To encourage continued work, strong public leadership must support discovery and development through the provision of stable and equitable pricing, together with stable and uniform regulatory guidelines. Only in this way will we be able to sustain the efforts required to keep us ahead of the fourth horseman.

\footnotetext{
1. Di Masi, J. A. et al. The price of innovation: new estimates of drug development costs. J. Health Econ. 22, 151-185 (2003).

2. Projan, S. J. Why is big Pharma getting out of antibacterial drug discovery? Curr. Opin. Microbiol. 6, 427-430 (2003).
} 